\title{
Agradecimento aos revisores "ad hoc"
}

\author{
Cristina Muccioli, Harley E. A. Bicas, Mauro Campos, Mauro Goldchmit, Paulo E. C. Dantas, \\ Samir J. Bechara, Vital Paulino Costa
}

\begin{abstract}
A Editoria Científica dos Arquivos Brasileiros de Oftalmologia agradece o trabalho competente dos colaboradores listados abaixo, na análise dos artigos do ano de 2006, imprescindível na manutenção do adequado nível científico e dentro dos mais rigorosos padrões do processo de revisão aos pares.
\end{abstract}

The Scientific Editorial Board from the Arquivos Brasileiros de Oftalmologia thanks the competent job of ours collaborators listed bellow on the peer review process in 2006, your careful evaluations of prospective articles help this journal maintain a high standard in publication of peer reviewed articles in Ophthalmology.
A. Duarte
Acácio Alves de Souza Lima Filho
Acácio Muralha Neto
Adriana Berezovsky
Adriana dos Santos Forseto
Áisa Haidar Lani
Amélia Kamegasawa
Ana Estela Besteti Pires Ponce Sant'Anna
André Correia Maia de Carvalho
André M.Vieira Messias
André Marcelo Vieira Gomes
André Romano
Andréa Araújo Zin
Arnaldo Furman Bordon
Belquiz Rodrigues A. Nassaralla
Breno Barth
Bruno Castelo Branco
Carlos Alexandre de Amorim Garcia
Carlos Eduardo Leite Arieta
Carlos Teixeira Brandt
César Lipener
Christiane Rolim de Moura
Cláudio Renato Garcia
Clélia Maria Erwenne
Cleusa Coral Ghanem
Cristina Maria Bittencourt Garrido
Davi Araf
Denise de Vuono Chinzon
Denise Fornazari de Oliveira
Diane Ruschel Marinho

Edison João Geraissate Filho

Edmundo Frota de Almeida Sobrinho

Edson Procianoy

Edson Shizuo Mori

Eduardo Ferrari Marback

Eduardo Melani Rocha

Eduardo Minelli

Eduardo Sone Soriano

Élcio Hideo Sato

Elcio Roque Kleinpaul

Eliana Aparecida Forno

Enyr Saran Arcieri

Eric Pinheiro de Andrade

Érika Alessandra Silvino Rodrigues

Eurípedes Mota Moura

Fábio Ejzenbaum

Fábio Henrique Cacho Casanova

Fausto Uno

Felício Aristóteles da Silva

Fernanda Bon Duarte

Fernando César Abib

Flávio Jaime Rocha

Flávio Rocha Lima Paranhos

Francisco Assis Cordeiro Barbosa

Francisco Porfírio Neto Jr.

Galton Carvalho Vasconcelos

Geraldo de Barros Ribeiro

Gustavo Victor

Helia Soares Angotti

Italo Mundialino Marcon

Jacob Cohen

Jair Giampani Jr.

João Antonio Prata Junior

João Carlos Miranda Gonçalves

João J. Nassaralla Jr.

Joelice dos Santos Araújo

Jonathan Clive Lake

José Américo Bonatti

José Paulo Cabral Vasconcellos

José Vital Filho

Joyce Hisae Yamamoto 
Juliana Maria Ferraz Sallum

Laurentino Biccas Neto

Leiria de Andrade Neto

Leopoldo Magacho

Luciana Peixoto Finamor

Luciene Barbosa de Sousa

Luciene Chaves Fernandes

Luís Antonio Vieira

Luís Carlos Ferreira de Sá

Luís Eduardo Morato Rebouças de Carvalho

Luís Paves

Luiz Alberto Melo

Magno Antonio Ferreira

Maira Saad de Ávila Morales

Marcelo Carvalho da Cunha

Marcelo Francisco Gaal Vadas

Marco Aurélio Lana Peixoto

Marco Cezar Helena

Marcos Carvalho da Cunha

Marcos Wilson Sampaio

Maria Aparecida Onuki Haddad

Maria Auxiliadora M. Frazão Sibinelli

Maria Cristina Martins

Maria de Lourdes Motta Moreira Villas Boas

Maria do Carmo Pinheiro

Maria Kiyoko Oyamada

Maria Regina Catai Chalita

Maria Rosa Bet de Moraes e Silva

Maria Teresa Bonanomi

Mário Teruo Sato

Marivaldo Castro de Oliveira

Martha Maria Motono Chojniak

Maurício Bastos Pereira

Maurício Della Paolera

Mauro Nishi

Mauro Waiswol

Milton Massato Hida

Mirian Skaf

Mônica Fialho Cronemberger

Moysés Zajdenweber

Nelson Alexandre Sabrosa

Nilo Holzchuh
Nilva Simeren B. Moraes

Niro Kasahara

Norma Allemann

Norma Helen Medina

Patrícia Ioschpe Gus

Paulo Gelmann Vaidergorn

Paulo Schor

Renato Ambrósio Jr.

Ricardo Lewinsky

Ricardo Suzuki

Ricardo Themudo Lessa Waetge

Riuitiro Yamane

Roberto Freda

Roberto Lauande Pimentel

Roberto Pedrosa Galvão Filho

Roberto Pinto Coelho

Rodrigo Jorge

Ronaldo Boaventura Barcellos

Rosa Maria Graziano

Rosana Nogueira Pires da Cunha

Rosane Cruz Ferreira

Rui Barroso Schimiti

Ruth Miyuki Santo

Ruth Rosenhek Schor

Saly Bugmann Moreira

Samuel Rymer

Sandra Maria Canelas Beer

Sergio Burnier

Sergio Felberg

Sidney Julio de Faria e Sousa

Silvia Veitzman

Simone Finzi

Simone H. D. Von Faber Bison

Solange Rios Salomão

Vera Lucia D. Monte Mascaro

Vera Regina Cardoso Castanheira

Victor Evangelista de Faria Ferraz

Vinicius Coral Ghanem

Virgílio Centurion

Walter Yukihiko Takahashi

Wesley Ribeiro Campos

Wilmar Roberto Silvino

Zélia Maria da Silva Corrêa 